\title{
PAPER
}

\section{Seizure outcome after epilepsy surgery in patients with normal preoperative MRI}

\author{
K Chapman, E Wyllie, I Najm, P Ruggieri, W Bingaman, J Lüders, P Kotagal, D Lachhwani, \\ D Dinner, H O Lüders
}

J Neurol Neurosurg Psychiatry 2005;76:710-713. doi: 10.1136/jnnp.2003.026757

\begin{abstract}
See end of article for authors' affiliations

.....................

Correspondence to: Dr Elaine Wyllie, The Cleveland Clinic Foundation, 9500 Euclid Avenue, Cleveland, $\mathrm{OH}$ 44195, USA; wylliee@ccf. org
\end{abstract}

Received 26 August 2003 Revised version received 27 August 2004 Accepted 30 August 2004

\begin{abstract}
Objective: To determine outcome after epilepsy surgery in patients with normal preoperative magnetic resonance imaging (MRI).

Methods: 24 adult and paediatric patients with normal preoperative MRIs were studied. They underwent epilepsy surgery between 1994 and 2001 and had at least one year of follow up.

Results: At the most recent follow up, nine patients $(37 \%)$ were seizure-free and $18(75 \%)$ had at least a $90 \%$ reduction in seizure frequency with weekly or monthly seizures. Seizure freedom was not significantly different after resections in frontal (5/9) or temporal regions $(4 / 13)(p=0.24$, Fisher's exact test), or among patients with or without localising features on EEG, PET, or ictal SPECT. Subdural grids, used in 15 of 24 patients, helped tailor resections but were not associated with differences in outcome. Histopathology showed cortical dysplasia in 10 patients (42\%), non-specific findings in $13(54 \%)$, and hippocampal sclerosis in one (4\%). Cortical dysplasia was seen in seven patients with frontal resection $(78 \%)$ and non-specific findings in nine $(69 \%)$ with temporal resection. Seizure outcome did not differ on the basis of location of resection or histopathology.

Conclusions: While these results were less favourable than expected for patients with focal epileptogenic lesions seen on MRI, they represented worthwhile improvement for this patient population with high preoperative seizure burden. In this highly selected group, no single test or combination of tests further predicted postoperative seizure outcome.
\end{abstract}

$M$ agnetic resonance imaging (MRI) has become indispensable in the presurgical evaluation of patients with intractable epilepsy. Identification of a focal epileptogenic lesion on preoperative MRI may affect postoperative seizure outcome favourably in both adult and paediatric patients, depending on location and pathology. ${ }^{1-9}$ Similarly, other studies have shown a worse postoperative seizure outcome when no lesion has been found on MRI. ${ }^{10-17}$ As MRI technology improves, smaller and more subtle lesions are being imaged and identified. For example, the advent of FLAIR (fluid attenuation recovery) imaging has improved the detection of mesial temporal sclerosis and malformations of cortical development. ${ }^{7}{ }^{18-22}$ However, some patients continue to have no detectable lesions on MRI, despite the suggestion of a focal lesion on seizure semiology and neurophysiology. The purpose of this retrospective study was to review the outcome of epilepsy surgery in patients with drug resistant focal epilepsy and normal preoperative MRI.

\section{METHODS}

Adult and paediatric patients were identified from the Cleveland Clinic epilepsy surgery database if they had epilepsy surgery from August 1994 to 2001, normal preoperative MRI as interpreted by staff neuroradiologists, and at least one year of postoperative follow up. Only cases operated on after August 1994 were selected, because since 1994 all presurgical cases have had MRI investigation, which included the modern imaging techniques outlined below.

All MRI studies were carried out using either a $1.5 \mathrm{~T}$ or a $3 \mathrm{~T}$ magnetic resonance scanner (Siemens, Erlangen, Germany). Preoperative MRI for patients in whom temporal lobe pathology was suspected included Tl sagittal, MPRAGE (magnetisation prepared rapid gradient echo) coronal, fast FLAIR coronal, and fast T2 coronal MRI sequences.
Preoperative MRI for patients in which extratemporal pathology was suspected included fast FLAIR axial, fast T2 axial, TI sagittal, and MPRAGE coronal MRI sequences. The Tl images were acquired at $5 \mathrm{~mm}$ thickness with a $2 \mathrm{~mm}$ interslice gap, while T2 images were acquired using $6 \mathrm{~mm}$ thickness and a $2 \mathrm{~mm}$ interslice gap. All MPRAGE images were acquired as contiguous $2 \mathrm{~mm}$ thick slices. For the purposes of this study, MRIs were analysed by two reviewers (KC, IN) who were blinded to all clinical details. A blinded neuroradiologist (PR) interpreted any controversial MRIs. Patients were excluded because of identified MRI lesions such as neoplasia, hippocampal sclerosis, or vascular malformations. Patients with subtle MRI findings such as blurring of the grey-white matter junction were also excluded. Patients were not excluded if the only finding on visual analysis was mild sulcal variation or equivocally thickened cortex.

Results from video EEG, extraoperative subdural electrocorticography, positron emission tomography (PET), ictal single photon emission computed tomography (SPECT), and histopathology were obtained from the medical records. Patients were contacted by phone for information regarding seizure outcome following surgery. Seizure outcome was assessed at 6, 12, and 24 months after surgery and at the latest follow up, and was classified according to the system proposed by Wieser $e^{2} a^{23}$ (fig 1). Statistical significance was assessed using Fisher's exact test, with significance defined as a probability $(\mathrm{p})$ value of $<0.05$.

The study was approved by the institutional review board of the Cleveland Clinic Foundation.

Abbreviations: FLAIR, fluid attenuation recovery; MPRAGE, magnetisation prepared rapid gradient echo; SPECT, single photon emission computed tomography 


\begin{tabular}{|llll}
\hline \multicolumn{3}{l}{ Table 1} & \multicolumn{3}{l}{ Histopathology of resected tissue } \\
\hline Histopathology & $\begin{array}{l}\text { Cortical } \\
\text { dysplasia }\end{array}$ & Non-specific ${ }^{*}+$ & $\begin{array}{l}\text { Hippocampal } \\
\text { sclerosis }\end{array}$ \\
\hline Total $(n=24)$ & $10(42 \%)$ & $13(54 \%)$ & $1(4 \%)$ \\
Frontal $(n=9)$ & $7(78 \%)$ & $2(22 \%)$ & 0 \\
Temporal $\left.\right|^{*}(n=13)$ & $2(15 \%)$ & $9(69 \%)$ & $1(8 \%)$ \\
Central $(n=1)$ & $1(100 \%)$ & 0 & 0 \\
Multilobar $(n=1)$ & 0 & $1(100 \%)$ & 0 \\
\hline
\end{tabular}

*One temporal lobe specimen was normal.

tGliosis, heterotopia, chronic inflammation, and microinfarct.

\section{RESULTS}

\section{Patient demographics}

Of 823 patients who had epilepsy surgery during the specified time period, only $24(2.9 \%)$ satisfied the inclusion criteria. Their ages ranged from 2 to 49 years (median 18). Preoperative seizure burden was high: 15 patients (63\%) had daily seizures (five $(21 \%)$ having more than 10 seizures a day) and the remainder had at least weekly seizures. In all cases, the seizure symptomatology was consistent with the suspected seizure onset zone, as identified by interictal and ictal scalp EEG. The mean duration of epilepsy was 10 years, with a mean of six antiepileptic drugs used before surgery. The length of postoperative follow up was 12 to 60 months (mean 29). No unexpected surgical complications were encountered in our series.

\section{Histopathology}

Table 1 shows the histopathology of the resected tissue based on the site of resection. All but two patients had frontal or temporal lobe resections. Cortical dysplasia was seen in seven of nine specimens from the frontal lobe, while non-specific findings-including gliosis, neuronal heterotopia, chronic inflammation, and infarction-were observed in nine of 13 specimens from patients who had temporal lobe resections. A single patient had hippocampal sclerosis identified on histopathology but not on preoperative MRI.

\section{Seizure outcome}

Seizure outcome at different postoperative follow up intervals is shown in fig 1, and at the latest outcome (mean 29 months) in table 2 . At latest follow up, 18 patients (75\%) had at least a $90 \%$ reduction in seizures and nine $(37 \%)$ were seizure-free. Patients with more than $90 \%$ seizure reduction continued to have a seizure frequency ranging between one to two a week to one a month.

Two patients underwent repeat surgery to extend their resections. One of these became seizure-free at 18 months of follow up, and another patient had no change in her seizure frequency. Outcomes were classified after the second resection.

Seizure outcome for the group did not change significantly between different follow up intervals, so the latest available follow up data were used for other analyses. Results were expressed for two subsets: seizure-free patients had class 1 outcome, while patients with more than $90 \%$ seizure reduction had outcome in classes 1 to 4 (that is, seizurefree, with auras only, with one to three seizure days a year, or with more than $90 \%$ seizure reduction).

Of the nine patients who were seizure-free, three remained seizure-free after antiepileptic drugs were discontinued. Five of the remaining patients were seizure-free on single drug treatment.

All the patients were selected for surgery because they had strong scalp EEG and clinical evidence of focal epileptogenicity in the resected region. Table 3 shows the seizure outcome based on the presence of focal abnormalities within the area of resection on various tests. None of the patients had focal

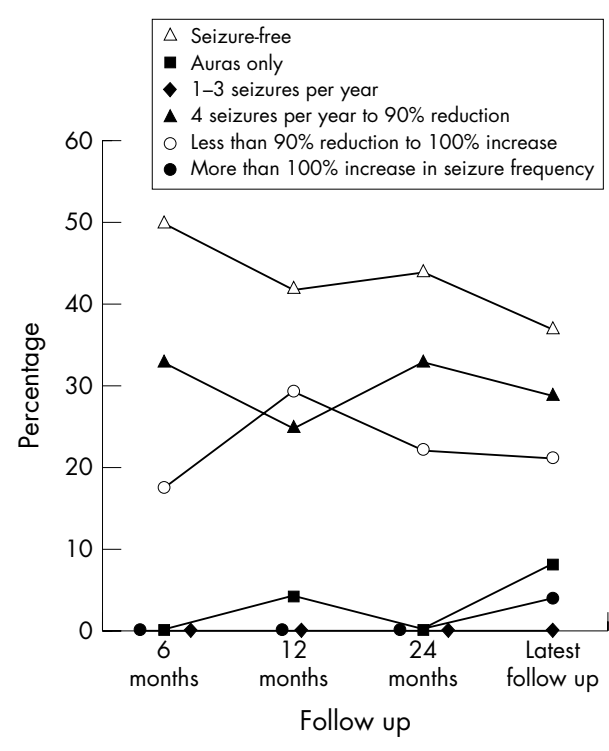

Figure 1 Seizure outcome in the whole cohort.

scalp EEG seizures arising from regions outside the resected area or from the contralateral hemisphere. One patient had a less well localised ictal EEG, but all the interictal sharp waves were localised to the resected region. Overall, 18 patients (75\%) had more than $90 \%$ of the interictal sharp waves on the scalp EEG maximal in the region that was resected; six of these were seizure-free and another nine had persistent seizures but more than $90 \%$ reduction in their seizure frequency; thus $15(83 \%)$ had more than $90 \%$ reduction in seizures. The remaining six patients had more than $10 \%$ of the interictal sharp waves outside the resected region; three of these had more than $90 \%$ seizure reduction (all were seizure-free).

Fifteen patients (63\%) also had prolonged extraoperative electrocorticography with subdural electrodes. Among 11 patients with focal interictal and ictal epileptiform discharges maximal in the resected region, four were seizure-free and eight had a more than $90 \%$ seizure reduction. Among four patients with more diffuse findings on subdural EEG, or with ictal or interictal epileptiform discharges maximal outside the resected region, one was seizure-free and one had persistent seizures but with more than $90 \%$ reduction in seizure frequency.

Among 16 patients in whom preoperative PET showed a single focal zone of hypometabolism maximal in the region of the resection, five were seizure-free and 12 had more than $90 \%$ seizure reduction. Among eight patients with normal, equivocal, or diffuse findings on PET, or with areas of hypometabolism distant from the resected region, four were seizure-free and six had more than $90 \%$ seizure reduction.

Among 13 patients whose preoperative ictal SPECT showed a single focal zone of hyperperfusion maximal in the resected region, seven were seizure-free and 10 had more than $90 \%$ seizure reduction. Two of five patients with normal, equivocal, or diffuse findings on ictal SPECT, or areas of hyperperfusion distant to the resected lesion, were seizurefree and four had more than $90 \%$ seizure reduction.

Twelve patients had strong evidence from both scalp EEG and PET of a single epileptogenic zone within the resected region, as defined above. Three of these were seizure-free and eight had more than $90 \%$ seizure reduction. Four of these patients also had findings on ictal SPECT that were localised to the region for resection: two with strongly localised and convergent findings on scalp EEG, PET, and ictal SPECT were seizure-free and all had more than $90 \%$ seizure reduction after surgery. 
Table 2 Latest outcome after surgical resection of all patients (mean 29 months)

\begin{tabular}{lllllll}
\hline & 1 & 2 & 3 & $\begin{array}{l}4 \\
(1-3 \text { seizures } \\
\text { a year) }\end{array}$ & $\begin{array}{l}\mathbf{4} \text { seizures a year } \\
\text { to } 90 \% \text { reduction) }\end{array}$ & $\begin{array}{l}5 \\
\text { (less than } 90 \% \text { reduction } \\
\text { to } 100 \% \text { increase) }\end{array}$ \\
\hline Total $(n=24)$ & $9(37 \%)$ & $2(8 \%)$ & 0 & $7(29 \%)$ & $5(21 \%)$ & $\begin{array}{l}6 \\
\text { (more than } 100 \% \text { increase } \\
\text { in seizure frequency) }\end{array}$ \\
Frontal lobe $(n=9)$ & $5(56 \%)$ & 0 & 0 & $1(11 \%)$ & $3(33 \%)$ & $1(4 \%)$ \\
Temporal lobe $(n=13)$ & $4(31 \%)$ & $2(15 \%)$ & 0 & $5(38 \%)$ & $2(15 \%)$ & 0 \\
Central $(n=1)$ & 0 & 0 & 0 & $1(100 \%)$ & 0 & 0 \\
Multilobar lobe $(n=1)$ & 0 & 0 & 0 & 0 & 0 & $1(100 \%)$ \\
\hline
\end{tabular}

Among 10 patients with cortical dysplasia identified on histopathology, five were seizure-free and eight had more than $90 \%$ seizure reduction. Among 12 patients with nonspecific findings on histopathology including gliosis, neuronal heterotopia, chronic inflammation, infarct, or hamartia, three were seizure-free and eight had more than $90 \%$ seizure reduction. One patient with hippocampal sclerosis was seizure-free, and one with normal histopathology had more than $90 \%$ seizure reduction.

There were no statistically significant differences in seizure outcome as assessed by Fisher's exact test.

\section{DISCUSSION}

In our series of patients with medically refractory focal epilepsy and normal preoperative MRI, 37\% were free of seizures after resective surgery and $75 \%$ overall had at least $90 \%$ reduction to weekly or monthly seizures. Although these results were less favourable than expected for patients with focal epileptogenic lesions seen on MRI, ${ }^{2}$ they represent an important improvement for this patient population with a very high preoperative seizure burden and severe medical intractability. The percentage of patients who were seizure-free at the latest follow up (median 28 months) was lower than at six months, one year, or two years postoperatively, although differences were not significant. The reduction in seizure freedom was mainly because two patients developed auras.

Previous studies have suggested that the absence of a lesion on preoperative MRI is a risk factor for persistent seizures after epilepsy surgery. In an actuarial study ${ }^{11}$ following temporal resection, only $29 \%$ of 24 patients who had normal preoperative MRI were free of seizures at a mean of 45 months after surgery, compared with $61 \%$ of 111 patients with a temporal lesion seen on preoperative MRI. In a series limited to frontal resections, ${ }^{12}$ only five of 17 patients with normal preoperative MRI were free of seizures after at least one year of follow up, compared with 21 of 32 patients $(66 \%)$ with a lesion seen on preoperative MRI. In contrast, in another series of patients who had temporal or extratemporal resection, ${ }^{13}$ no significant differences were reported between those with and without a lesion on preoperative MRI. One factor in that study may have been the use of less sensitive MRI techniques in the $60 \%$ of patients who were operated on before 1992. Other series have shown variable rates of seizure-free outcome after epilepsy surgery in patients with normal or non-localised preoperative MRI, compared with patients with focal lesions. ${ }^{14} 17$

Others have reported postoperative freedom from seizures for $68 \%$ to $89 \%$ of patients with neoplasms $s^{2-513} 24$ and for $67 \%$ to $91 \%$ of those with hippocampal sclerosis. ${ }^{2489}$ These rates appear higher than those in this and other ${ }^{11-13}$ series of patients without a focal lesion seen on preoperative MRI, and probably reflect the more focal nature of these lesions. Our results are closer to those reported for patients with malformations of cortical development seen on preoperative MRI, with $42 \%$ to $52 \%$ of patients seizure-free in some series. $^{2}{ }^{71325}$ This similarity to our results may reflect the $42 \%$ of patients in our series who had occult cortical dysplasia diagnosed by histopathology. Our patients had more diffuse lesions with less clearly defined margins than those seen in the setting of focal lesions, such as hippocampal sclerosis, and this may explain our lower rates of seizure freedom.

The highly selected nature of our patients with normal preoperative MRIs is clear from their small number, comprising only $2.9 \%$ of all patients who had epilepsy surgery at our institution during the specified period. They were all selected for surgery-despite a normal preoperative MRIbecause of a high preoperative seizure burden and strong EEG and clinical features suggesting a relatively limited, resectable focal epileptogenic zone. None of the patients had scalp EEG seizures arising from regions outside the resected areas, and some of the patients also had strongly localising features on PET, ictal SPECT, and subdural EEG. Within this small, highly selected series, however, no single test or combination of tests clearly further predicted favourable or poor postoperative seizure outcome.

In our series of patients with normal preoperative MRI, PET and ictal SPECT results were concordant with scalp EEG localisation in $50 \%$ and $39 \%$ of cases, respectively. Others have explored the concordance rate between EEG and PET or ictal SPECT in patients with normal MRI. In one series, ictal

Table 3 Seizure outcome based on focal abnormalities in the area of resection detected by different testing methods

\begin{tabular}{lllll}
\hline \multirow{2}{*}{$\begin{array}{l}\text { Focal abnormalities in area of } \\
\text { resection }\end{array}$} & & \multicolumn{3}{l}{ Persistent seizures } \\
\cline { 4 - 5 } & & Seizure-free & $>\mathbf{9 0 \%}$ reduction & $<90 \%$ reduction \\
\hline Scalp EEG & Yes & $6(33 \%)$ & $9(50 \%)$ & $3(17 \%)$ \\
& No & $3(50 \%)$ & 0 & $3(50 \%)$ \\
Invasive EEG & Yes & $4(36 \%)$ & $4(36 \%)$ & $3(27 \%)$ \\
& No & $1(25 \%)$ & $1(25 \%)$ & $2(50 \%)$ \\
PET & Yes & $5(31 \%)$ & $7(44 \%)$ & $2(25 \%)$ \\
& No & $4(50 \%)$ & $2(25 \%)$ & $3(23 \%)$ \\
Ictal SPECT & Yes & $7(54 \%)$ & $3(23 \%)$ & $1(10 \%)$ \\
& No & $2(40 \%)$ & $2(40 \%)$ & \\
\hline
\end{tabular}

None of the differences was statistically significant.

$\mathrm{PET}$, positron emission tomography; SPECT, single photon emission computed tomography. 
SPECT was concordant with EEG in $93 \%$ of patients with temporal lobe epilepsy, and in only $56 \%$ of patients with extratemporal epilepsy. ${ }^{26}$ Others noted similar results for PET in patients with non-lesional temporal or extratemporal epilepsy. ${ }^{15}$ Studies of concordance with EEG, however, do not address the clinical question of a test's independent predictive value for a seizure-free postoperative outcome. In our series, PET and ictal SPECT results were usually concordant with EEG localisation, but concordant results did not appear to enhance significantly the likelihood of a favourable postoperative outcome.

Histopathology revealed cortical dysplasia in $78 \%$ of our frontal lobe specimens, and less specific findings (gliosis, neuronal heterotopia, chronic inflammation, microinfarction, and hamartia) in $69 \%$ of the temporal lobe specimens. However, seizure outcome did not differ significantly between groups based on resection location or histopathology. Only a single MRI negative patient in this series had hippocampal neuronal loss and gliosis suggesting hippocampal sclerosis, and he remained seizure-free following resection. Other studies of temporal lobe epilepsy with normal preoperative MRI have also shown that hippocampal sclerosis is rarely found to be the pathological substrate..$^{10} 1727$

After careful evaluation, each of the MRIs in our series was normal, despite having histologically abnormal specimens. This suggests that our current MRI technology may be ineffective in detecting small or subtle lesions. As technology improves and new techniques are discovered, fewer patients with focal epilepsy and normal MRIs may be found.

Many investigators have emphasised the importance of using subdural electrodes in the evaluation of patients with non-lesional, particularly extratemporal, epilepsy. ${ }^{328} 29$ Previous electrocorticographic studies have demonstrated the value of a stable resectable ictal onset zone for the best prognosis for seizure freedom. ${ }^{13}{ }^{30-33}$ Invasive studies with subdural electrodes were done in $63 \%$ of our patients, typically in complicated cases requiring a clear definition of the epileptogenic zone in relation to functional cortex. In our limited number of study patients, no outcome differences were identified between patients with or without subdural evaluation. However, in individual cases the invasive testing may have helped to devise the surgical strategy.

In summary, epilepsy surgery may be an appropriate option, despite normal MRI, in patients with a high preoperative seizure burden and strong EEG and clinical evidence of a focal resectable epileptogenic zone. The likelihood of postoperative seizure freedom in our series was relatively low (37\%), but $75 \%$ of patients had at least a $90 \%$ reduction in seizure frequency. In patients with severe, medically refractory epilepsy, this improvement may be very worthwhile. Larger prospective studies will be required to further characterise this important surgical subgroup.

\section{Authors' affiliations}

K Chapman, Department of Neurology, Baylor College of Medicine, Houston, Texas, USA

E Wyllie, I Najm, P Kotagal, D Lachhwani, D Dinner, H O Lüders, Departments of Neurology, The Cleveland Clinic Foundation, Cleveland, Ohio, USA

W Bingaman, J Lüders, Departments of Neurosurgery, The Cleveland Clinic Foundation

P Ruggieri, Departments of Neuroradiology, The Cleveland Clinic Foundation

Competing interests: none declared

\section{REFERENCES}

1 Engel J, Van Ness P, Rasmussen T, et al. Outcome with respect to epileptic seizures. In: Engel J, ed. Surgical treatment of the epilepsies, 2 nd ed. New York: Raven Press, 1993:609-21
2 Wyllie E, Comair YG, Kotagal P, et al. Seizure outcome after epilepsy surgery in children and adolescents. Ann Neurol 1998;44:740-8.

3 Zentner J, Hufnagel A, Ostertun B, et al. Surgical treatment of extratemporal epilepsy: clinical, radiologic, and histopathologic findings in 60 patients. Epilepsia 1996;37:1072-80.

4 Zentner J, Hufnagel A, Wolf HK, et al. Surgical treatment of temporal lobe epilepsy: clinical, radiological, and histopathological findings in 178 patients. J Neurol Neurosurg Psychiatry 1995;58:666-73.

5 Schramm J, Kral T, Grunwald T, et al. Surgical treatment for neocortical temporal lobe epilepsy: clinical and surgical aspects and seizure outcome. J Neurosurg 2001;94:33-42.

6 Patel H, Garg BP, Salanova V, et al. Tumor-related epilepsy in children. J Child Neurol 2001;16:141-5.

7 Edwards JC, Wyllie E, Ruggeri PM, et al. Seizure outcome after surgery for epilepsy due to malformation of cortical development. Neurology 2000:55:1110-14.

8 Kuzniecky R, Ho SS, Martin R, et al. Temporal lobe developmental malformations and hippocampal sclerosis: epilepsy surgical outcome. Neurology 1999:479-84.

9 Mohamed A, Wyllie E, Ruggieri P, et al. Temporal lobe epilepsy due to hippocampal sclerosis in pediatric candidates for epilepsy surgery. Neurology 2001;56: 1643-9.

10 Siegel AM, Jobst BC, Thadani VM, et al. Medically intractable, localizationrelated epilepsy with normal MRI: presurgical evaluation and surgical outcome in 43 patients. Epilepsia 2001;42:883-8.

11 Berkovic SF, Mclntosh AM, Kalnins RM, et al. Preoperative MRI predicts outcome of temporal lobectomy: an actuarial analysis. Neurology 1995;45:1358-63.

12 Smith JR, Lee MR, King DW, et al. Results of lesional vs. nonlesional frontal lobe epilepsy surgery. Stereotact Funct Neurosurg 1997;69:202-9.

13 Paolicchi JM, Jayakar P, Dean P, et al. Predictors of outcome in pediatric epilepsy surgery. Neurology 2000;54:642-7.

14 Cukiert A, Buratini JA, Machado E, et al. Results of surgery in patients with refractory extratemporal epilepsy with normal or nonlocalizing magnetic resonance findings investigated with subdural grids. Epilepsia 2001:42:889-94.

15 Won HJ, Chang KH, Cheon JE, et al. Comparison of MR imaging with PET and ictal SPECT in 118 patients with intractable epilepsy. Am J Neuroradiol 1999;20:593-9.

16 Park SA, Lim SR, Kim GS, et al. Ictal electrocorticographic findings related with surgical outcomes in nonlesional neocortical epilepsy. Epilepsy Res 2002;48:199-206.

17 Holmes MD, Born DE, Kutsy RL, et al. Outcome after surgery in patients with refractory temporal lobe epilepsy and normal MRI. Seizure 2000;9:407-11.

18 Lenclos N, Oppenheim C, Dormont D, et al. [MRI of drug-resistant epilepsies: contribution of FLAIR sequence in a series of 150 patients]. J Neuroradiol 2000;27:164-72

19 Usui N, Matsuda K, Mihara T, et al. MRI of cortical dysplasia - correlation with pathological findings. Neuroradiology 2001;43:830-7.

20 Marusic P, Najm IM, Ying Z, et al. Focal cortical dysplasias in eloquent cortex: functional characteristics and correlation with MRI and histopathologic changes. Epilepsia 2002;43:27-32.

21 Diehl B, Najm I, Mohamed A, et al. Fluid-attenuated inversion recovery: correlations of hippocampal cell densities with signal abnormalities. Neurology 2001;57:1029-32.

22 Jack CR, Rydberg $\mathrm{CH}$, Krecke KN, et al. Mesial temporal sclerosis: diagnosis with fluid-attenuated inversion- recovery versus spin-echo MR imaging. Radiology 1996; 199:367-73.

23 Wieser HG, Blume WT, Fish D, et al. ILAE Commission report. Proposal for a new classification of outcome with respect to epileptic seizures following epilepsy surgery. Epilepsia 2001;42:282-6.

24 Rossi GF, Colicchio G, Scerrati M. Resection surgery for partial epilepsy. Relation of surgical outcome with some aspects of the epileptogenic process and surgical approach. Acta Neurochir (Wien) 1994;130:101-10.

25 Kloss S, Pieper T, Pannek H, et al. Epilepsy surgery in children with focal cortical dysplasia (FCD): results of long-term seizure outcome. Neuropediatrics 2002;33:21-6.

26 Spencer SS. The relative contributions of MRI, SPECT, and PET imaging in epilepsy. Epilepsia 1994;35(suppl 6):S72-89.

27 Lee DH, Gao FQ, Rogers JM, et al. MR in temporal lobe epilepsy: analysis with pathologic confirmation. Am J Neuroradiol 1998;19:19-27.

28 Adelson PD, O'Rourke DK, Albright AL. Chronic invasive monitoring for identifying seizure foci in children. Neurosurg Clin North Am 1995;6:491-504.

29 Jayakar P, Duchowny M, Resnick TJ. Subdural monitoring in the evaluation of children for epilepsy surgery. J Child Neurol 1994;9(suppl 2):61-6.

30 Hufnagel A, Elger CE, Pels $\mathrm{H}$, et al. Prognostic significance of ictal and interictal epileptiform activity in temporal lobe epilepsy. Epilepsia 1994;35: 1146-53.

31 Jung WY, Pacia SV, Devinsky R. Neocortical temporal lobe epilepsy: intracranial EEG features and surgical outcome. J Clin Neurophysiol 1999; 16:419-25.

32 Quesney LF. Extratemporal epilepsy: clinical presentation, pre-operative EEG localization and surgical outcome. Acta Neurol Scand Suppl 1992;140:81-94.

33 Henry TR, Ross DA, Schuh LA, et al. Indications and outcome of ictal recording with intracerebral and subdural electrodes in refractory complex partial seizures. J Clin Neurophysiol 1999;16:426-38. 